International Journal of Pure and Applied Mathematics

Volume 93 No. 2 2014, 181-189

ISSN: 1311-8080 (printed version); ISSN: 1314-3395 (on-line version)

url: http://www.ijpam.eu

doi: http://dx.doi.org/10.12732/ijpam.v93i2.3

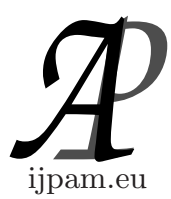

\title{
NORMALITY AND SHARING VALUES
}

\author{
Gopal Datt
}

Department of Mathematics

University of Delhi

Delhi 110007, INDIA

\begin{abstract}
In this Paper we prove some normality criteria for a family of holomorphic functions, where a complex value is shared by every function from the family and its $k^{t h}$ derivative. We use some results from Value Distribution Theory for proving the results.
\end{abstract}

AMS Subject Classification: 30D45, 30D35

Key Words: analytic functions, holomorphic functions, normal families, sharing values

\section{Introduction}

We denote the complex plane by $\mathbb{C}$, and the unit disk by $\Delta$. Let $f$ be a meromorphic function in $\mathbb{C}$. We say that $f$ is a normal function if there exits a positive $M$ such that $f^{\#}(z) \leq M$ for all $z \in \mathbb{C}$, where $f^{\#}=\frac{\left|f^{\prime}(z)\right|}{1+|f(z)|^{2}}$ denotes the spherical derivative of $f .\{[8]$, P. 171\}.

A family $\mathcal{F}$ of analytic functions on a domain $\Omega \subseteq \mathbb{C}$ is normal in $\Omega$ if every sequence of functions $\left\{f_{n}\right\} \subseteq \mathcal{F}$ contains either a subsequence which converges to a limit function $f \not \equiv \infty$ uniformly on each compact subset of $\Omega$, or a subsequence which converges uniformly to $\infty$ on each compact subset. $\{[10]$, P.33\}.

Received: November 2, 2013

(C) 2014 Academic Publications, Ltd. url: www.acadpubl.eu 
In this paper, we use the following standard notations of value distribution theory,

$$
T(r, f) ; m(r, f) ; N(r, f) ; \bar{N}(r, f), \ldots
$$

We denote $S(r, f)$ any function satisfying

$$
S(r, f)=o\{T(r, f)\}, \text { as } r \rightarrow+\infty,
$$

possibly outside of a set with finite measure.

According to Bloch's principle every condition which reduces a meromorphic function in the plane to a constant, makes the family of meromorphic functions in a domain $\mathcal{D}$ normal. Rubel gave four counter examples to Bloch principle.

Let $f$ and $g$ be meromorphic functions in a domain $D$ and $a \in \mathbb{C}$. Let zeros of $f-a$ are zeros of $g-a$ (ignoring multiplicity), we write $f=a \Rightarrow g=a$. Hence $f=a \Longleftrightarrow g=a$ means that $f-a$ and $g-a$ have the same zeros (ignoring multiplicity). If $f-a \Longleftrightarrow g-a$, then we say that $f$ and $g$ share the value $z=a \mathrm{IM} .\{[14], \mathrm{p} .108\}$.

Let us recall the following known results that establish connection between shared values and normality. Mues and Steinmetz proved the following result :

Theorem 1.1. [7] Let $f$ be a non-constant meromorphic function in the plane. If $f$ and $f^{\prime}$ share three distinct complex numbers $a_{1}, a_{2}, a_{3}$ then $f \equiv f^{\prime}$.

Wilhelm Schwick seems to have been the first to draw a connection between normality and shared values. He proved the following theorem :

Theorem 1.2. [11] Let $\mathcal{F}$ be a family of meromorphic functions on a domain $G$ and $a_{1}, a_{2}, a_{3}$ be distinct complex numbers. If $f$ and $f^{\prime}$ share $a_{1}$, $a_{2}, a_{3}$ for every $f \in \mathcal{F}$, then $\mathcal{F}$ is normal in $G$.

In 2000, Chen and Hua proved the following result :

Theorem 1.3. [1] Let $\mathcal{F}$ be a family of holomorphic functions in a domain D. Suppose that there exists a non zero $a \in \mathbb{C}$ such that for each function $f \in F ; f, f^{\prime}$ and $f^{\prime \prime}$ share the value $z=a I M$ in $D$. Then the family $\mathcal{F}$ is normal in $D$.

Fang and $\mathrm{Xu}$ improved theorem 1.1 and theorem 1.2 by proving the following theorems :

Theorem 1.4. [5] Let $\mathcal{F}$ be a family of holomorphic functions on a domain $D$ and let $a, b$ be two distinct finite complex numbers such that $b \neq 0$. If for any $f \in \mathcal{F}, f$ and $f^{\prime}$ share $z=a$ IM and $f(z)=b$ whenever $f^{\prime}(z)=b$ then $\mathcal{F}$ is normal in $D$. 
Theorem 1.5. [5] Let $\mathcal{F}$ be a family of holomorphic functions in a domain $D$, and let $a$ be a non zero finite complex number. If for any $f \in \mathcal{F} f$ and $f^{\prime}$ share $z=a$ IM and $f^{(k)}(z)=a, f^{(k+1)}(z)=a$ whenever $f(z)=a$. Then $\mathcal{F}$ is normal in $D$.

It is natural to consider: What can we say if $f^{\prime}$ in Theorem 1.4 and Theorem 1.5 is replaced by the $k$-th derivative $f^{(k)}$ ? In this paper, we prove the following results.

Theorem 1.6. Let $\mathcal{F}$ be a family of holomorphic functions on a domain $D$ such that all zeros of $f \in \mathcal{F}$ are of multiplicity at least $k$, where $k$ is a positive integer. Let $a, b$ be two distinct finite complex numbers such that $b \neq$ 0 . Suppose for any $f \in \mathcal{F}$ satisfies the following conditions

1. $f$ and $f^{(k)}$ share $z=a I M$

2. $f(z)=b$ whenever $f^{(k)}(z)=b$

Then $\mathcal{F}$ is normal in $D$.

Theorem 1.7. Let $\mathcal{F}$ be a family of holomorphic functions in a domain $D$ such that all zeros of $f \in \mathcal{F}$ are of multiplicity at least $k$, where $k$ is a positive integer and let a be a non zero finite complex number. If for any $f \in \mathcal{F} f$ and $f^{(k)}$ share $z=a$ IM and $f^{(k+1)}(z)=a$ whenever $f(z)=a$. Then $\mathcal{F}$ is normal in $D$.

Remark 1.8. The hypothesis $a \neq b$ can not be dropped in Theorem 1.6.

Example 1.9. Let $D=\Delta=\{z:|z|<1\}$, let $k=1$ and $a=b=1$, and

$$
\mathcal{F}=\left\{e^{n z}-\frac{1}{n^{k}}+1: n=1,2,3, \ldots\right\} .
$$

Then for any $f \in \mathcal{F}$, and

$$
f=e^{n z}-\frac{1}{n^{k}}+1, \quad f^{(k)}=n^{k} e^{n z} .
$$

Clearly, all other conditions of Theorem 1.6 are satisfied. However, $\mathcal{F}$ is not normal in $\Delta$. This example confirms that $b \neq 0$ is necessary in Theorem 1.6 as $f^{(k)}(z) \neq 0$.

Example 1.10. Let $D=\Delta=\{z:|z|<1\}$, let $k=1, a=\left((-1)^{k+1}+1\right) b$ and

$$
\mathcal{F}=\left\{b \frac{\left(z-\frac{1}{n}\right)^{k}}{k !}+\frac{(-1)^{k+1}}{k ! n\left(z-\frac{1}{n}\right)}+a: n=1,2,3, \ldots\right\} .
$$


Then, for every $f_{n}(z) \in \mathcal{F}$,

$$
f_{n}(z)=b \frac{\left(z-\frac{1}{n}\right)^{k}}{k !}+\frac{(-1)^{k+1}}{k ! n\left(z-\frac{1}{n}\right)}+a, \quad f_{n}^{(k)}(z)=b-\frac{1}{n\left(z-\frac{1}{n}\right)^{k+1}} .
$$

Clearly, $f_{n}$ and $f_{n}^{(k)}$ share $a$ and $f_{n}^{(k)}(z) \neq b$, so that $f_{n}(z)=b$ whenever $f_{n}^{(k)}(z)=b$. But $\mathcal{F}$ is not normal in $D$. This example shows that Theorem 1.6 is not valid for a family of meromorphic functions.

We will use the tools of Fang and $\mathrm{Xu}$ [5] which they used in their paper.

\section{Some Lemmas}

In order to prove our results we need the following Lemmas.

Lemma 2.1. [16] [9](Zalcman's lemma) Let $\mathcal{F}$ be a family of holomorphic functions in the unit disk $\Delta$, with the property that for every function $f \in \mathcal{F}$, the zeros of $f$ are of multiplicity at least $k$. If $\mathcal{F}$ is not normal at $z_{0}$ in $\Delta$, then for $0 \leq \alpha<k$, there exist

1. a sequence of complex numbers $z_{n} \rightarrow z_{0},\left|z_{n}\right|<r<1$,

2. a sequence of functions $f_{n} \in \mathcal{F}$,

3. a sequence of positive numbers $\rho_{n} \rightarrow 0$,

such that $g_{n}(\zeta)=\rho_{n}^{-\alpha} f_{n}\left(z_{n}+\rho_{n} \zeta\right)$ converges to a non-constant entire function $g$ on $\mathbb{C}$. Moreover $g$ is of order at most one. If $\mathcal{F}$ possesses the additional property that there exists $M>0$ such that $\left|f^{(k)}(z)\right| \leq M$ whenever $f(z)=0$ for any $f \in \mathcal{F}$, then we can take $\alpha=k$.

Lemma 2.2. [6] [15] Let $f$ be a non-constant meromorphic function. Then for $k \geq 1, b \neq 0, \infty$,

$$
T(r, f) \leq \bar{N}(r, f)+N\left(r, \frac{1}{f}\right)+N\left(r, \frac{1}{f^{(k)}-b}\right)-N\left(r, \frac{1}{f^{(k+1)}}\right)+S(r, f) .
$$

\section{Proof of Theorem 1.6}

Since normality is a local property, we assume that $D=\Delta=\{z:|z|<1\}$. Suppose $\mathcal{F}$ is not normal in $D$. Without loss of generality we assume that $\mathcal{F}$ is not normal at the point $z_{0}$ in $\Delta$. Then by Lemma 2.1 , there exist 
1. a sequence of complex numbers $z_{n} \rightarrow z_{0},\left|z_{n}\right|<r<1$,

2. a sequence of functions $f_{n} \in \mathcal{F}$ and

3. a sequence of positive numbers $\rho_{n} \rightarrow 0$,

such that $g_{n}(\zeta)=\rho_{n}^{-k}\left[f_{n}\left(z_{n}+\rho_{n} \zeta\right)-a\right]$ converges locally uniformly to a nonconstant entire function $g$. Moreover $g$ is of order at most one.

Now we claim that $g=0$ if and only if $g^{(k)}=a$ and $g^{(k)} \neq b$. Suppose $g\left(\zeta_{0}\right)=0$. Then by Hurwitz's theorem there exist $\zeta_{n} ; \zeta_{n} \rightarrow \zeta_{0}$ such that

$$
g_{n}\left(\zeta_{n}\right)=\rho_{n}^{-k}\left[f_{n}\left(z_{n}+\rho_{n} \zeta_{n}\right)-a\right]=0 .
$$

Thus $f_{n}\left(z_{n}+\rho_{n} \zeta_{n}\right)=a$. Since $f_{n}$ and $f_{n}^{(k)}$ share $z=a$ IM, we have

$$
g_{n}^{k}\left(\zeta_{n}\right)=f_{n}^{(k)}\left(z_{n}+\rho_{n} \zeta_{n}\right)=a .
$$

Hence

$$
g^{(k)}\left(\zeta_{0}\right)=\lim _{n \rightarrow \infty} g_{n}^{(k)}\left(\zeta_{n}\right)=a .
$$

Thus we have proved that $g^{(k)}=a$ whenever $g=0$.

On the other hand, if $g^{(k)}\left(\zeta_{0}\right)=a$, then there exist $\zeta_{n} ; \zeta_{n} \rightarrow \zeta_{0}$ such that $g_{n}^{(k)}\left(\zeta_{n}\right)=f_{n}^{(k)}\left(z_{n}+\rho_{n} \zeta_{n}\right)=a, n=1,2, \ldots$. Hence $f_{n}\left(z_{n}+\rho_{n} \zeta_{n}\right)=a$ and $g_{n}\left(\zeta_{n}\right)=0$ for $n=1,2, \ldots$. Thus

$$
g\left(\zeta_{0}\right)=\lim _{n \rightarrow \infty} g_{n}\left(\zeta_{n}\right)=0 .
$$

This shows that $g=0$ whenever $g^{(k)}=a$. Hence $g=0$ if and only if $g^{k}=a$.

Next, we prove $g^{(k)}(\zeta) \neq b$. Suppose there exist $\zeta_{0}$ satisfying $g^{(k)}\left(\zeta_{0}\right)=b$. Then, by Hurwitz's theorem, there exist a sequence $\zeta_{n} \rightarrow \zeta_{0}$ and $g_{n}^{(k)}\left(\zeta_{n}\right)=$ $b, n=1,2, \ldots$. Since $f_{n}(z)=b$ whenever $f_{n}^{(k)}(z)=b \Rightarrow f_{n}\left(z_{n}+\rho_{n} \zeta_{n}\right)=b$ and,

$$
g_{n}\left(\zeta_{n}\right)=\rho_{n}^{-k}\left[f_{n}\left(z_{n}+\rho_{n} \zeta_{n}\right)-a\right]=\rho_{n}^{(k)}[b-a] \rightarrow \infty,
$$

this contradicts

$$
\lim _{n \rightarrow \infty} g_{n}\left(\zeta_{n}\right)=g\left(\zeta_{0}\right) \neq \infty
$$

. So $g^{(k)}(\zeta) \neq b$. Hence we get,

$$
g^{(k)}(\zeta)=b+e^{A \zeta+B}
$$


where $A$ and $B$ are two constants. We claim that $A=0$. Suppose that $A \neq 0$, then

$$
g(\zeta)=\frac{b \zeta^{k}}{k !}+\frac{e^{A \zeta+B}}{A^{k}}+\frac{c_{1} \zeta^{k-1}}{(k-1) !}+\ldots+c_{k-1} \zeta+c_{k},
$$

where $c_{1}, c_{2}, \ldots, c_{k}$ are constants. Let $g^{(k)}=a$. Then by (1), (2) and $g(\zeta)=$ 0 wheneverg $g^{(k)}(\zeta)=a$, we have

$$
\frac{b \zeta^{k}}{k !}+\frac{c_{1} \zeta^{k-1}}{(k-1) !}+\ldots+c_{k}+\frac{b-a}{A^{k}}=0 .
$$

This is a polynomial of degree $k$ in $\zeta$ this polynomial has $k$ solutions. Which contradicts the fact that $g^{(k)}$ has infinitely many solutions. Thus we have,

$$
g^{(k)}(\zeta)=b+e^{B}
$$

and

$$
g(\zeta)=\left(b+e^{B}\right) \frac{\zeta^{k}}{k !}+\frac{c_{1} \zeta^{k-1}}{(k-1) !}+\ldots+c_{k}
$$

Since $g$ is non-constant, this contradicts $g(\zeta)=0 \Leftrightarrow g^{(k)}(\zeta)=a$. Thus $\mathcal{F}$ is normal in $D$. This completes the proof of theorem.

\section{Proof of Theorem 1.7}

Suppose $\mathcal{F}$ is not normal in $\Delta$; without loss of generality we assume that $\mathcal{F}$ is not normal at the point $z=0$. Then by Lemma 2.1, there exist

1. a sequence of complex numbers $z_{n} \rightarrow 0,\left|z_{n}\right|<r<1$,

2. a sequence of functions $f_{n} \in \mathcal{F}$ and

3. a sequence of positive numbers $\rho_{n} \rightarrow 0$,

such that $g_{n}(\zeta)=\rho_{n}^{-k}\left[f_{n}\left(z_{n}+\rho_{n} \zeta\right)-a\right]$ converges locally uniformly to a nonconstant entire function $g$. Moreover $g$ is of order at most one.

Now we claim that $g=0$ iff $g^{(k)}=a$ and $g^{(k+1)}=0$ whenever $g=0$. Let $g\left(\zeta_{0}\right)=0$. Then by Hurwitz's theorem there exist $\zeta_{n} ; \zeta_{n} \rightarrow \zeta_{0}$ such that

$$
g_{n}\left(\zeta_{n}\right)=\rho_{n}^{-k}\left[f_{n}\left(z_{n}+\rho_{n} \zeta_{n}\right)-a\right]=0 .
$$


Thus $f_{n}\left(z_{n}+\rho_{n} \zeta_{n}\right)=a$, since $f_{n}$ and $f_{n}^{(k)}$ share $z=a$ IM, we have

$$
g_{n}^{k}\left(\zeta_{n}\right)=f_{n}^{(k)}\left(z_{n}+\rho_{n} \zeta_{n}\right)=a
$$

and

$$
g_{n}^{(k+1)}\left(\zeta_{n}\right)=\rho_{n} f_{n}^{(k+1)}\left(z_{n}+\rho_{n} \zeta_{n}\right) .
$$

Which implies that

$$
g^{(k)}\left(\zeta_{0}\right)=\lim _{n \rightarrow \infty} g_{n}^{(k)}\left(\zeta_{n}\right)=a
$$

and

$$
g^{(k+1)}\left(\zeta_{0}\right)=\lim _{n \rightarrow \infty} g_{n}^{(k+1)}\left(\zeta_{n}\right)=0 .
$$

Thus we get, $g^{(k)}=a$ whenever $g=0$ and $g^{(k+1)}=0$ whenever $g=0$.

On then other hand, if $g^{(k)}\left(\zeta_{0}\right)=a$ then there exist $\zeta_{n} \rightarrow \zeta_{0}$ such that $g_{n}^{(k)}\left(\zeta_{n}\right)=f_{n}^{(k)}\left(z_{n}+\rho_{n} \zeta_{n}\right)=a, n=1,2, \ldots$. Hence $f_{n}\left(z_{n}+\rho_{n} \zeta_{n}\right)=a$ and $g_{n}\left(\zeta_{n}\right)=0$ for $n=1,2, \ldots$. Thus

$$
g\left(\zeta_{0}\right)=\lim _{n \rightarrow \infty} g_{n}\left(\zeta_{n}\right)=0 .
$$

This shows that $g=0$ whenever $g^{(k)}=a$. Hence $g=0$ if and only if $g^{k}=a$ and $g^{(k+1)}=0$ whenever $g=0$.

Now using Lemma 2.2 and Nevanlinna's first fundamental theorem, we have

$$
\begin{aligned}
T(r, g) & \leq \bar{N}(r, g)+N\left(r, \frac{1}{g}\right)+N\left(r, \frac{1}{g^{(k)}-a}\right)-N\left(r, \frac{1}{g^{(k+1)}}\right)+S(r, g) \\
& =N\left(r, \frac{1}{g}\right)+N\left(r, \frac{1}{g^{(k)}-a}\right)-N\left(r, \frac{1}{g^{(k+1)}}\right)+S(r, g) \\
& \leq N\left(r, \frac{1}{g^{(k)}-a}\right)-\bar{N}\left(r, \frac{1}{g^{(k+1)}}\right)+S(r, g) \\
& \leq T\left(r, \frac{1}{g^{(k)}-a}\right)-\bar{N}\left(r, \frac{1}{g^{(k+1)}}\right)+S(r, g) \\
& \leq T\left(r, g^{(k)}-a\right)-\bar{N}\left(r, \frac{1}{g^{(k+1)}}\right)+S(r, g) \\
& \leq T(r, g)-\bar{N}\left(r, \frac{1}{g^{(k+1)}}\right)+S(r, g) .
\end{aligned}
$$

Thus we get

$$
\bar{N}\left(r, \frac{1}{g^{(k+1)}}\right)=S(r, g),
$$

by (3), (4) and the claim $\left(g=0\right.$ if and only if $g^{(k)}=a, g^{(k+1)}=0$ whenever $g=$ 0 ) we get a contradiction: $T(r, g)=S(r, g)$. It proves the theorem. 


\section{References}

[1] H. H. Chen and X. H. Hua, Normal Values Concerning Shared Values, Israel Journal of Mathematics 115 (2000), 355-362, doi: 10.1007/BF02810596.

[2] J. F. Chen, Normal Families and Shared Sets of Meromorphic Functions, Rocky Mountain Journal of Mathematics, 41 (2011), no. 1, 37-43, doi: 10.1216/RMJ-2011-41-1-37.

[3] J. F. Chen, M. L. Fang, Normal Families And Shared Functions of Meromorphic Functions, Israel Journal of Mathematics 180 (2010), 129-142, doi: 10.1007/S11856-010-0097-7.

[4] M. L. Fang, Picard Values and Normality criterion, Bull. Korean Math. Soc. 38 (2001), No. 2, 379-387.

[5] M. L. Fang and Y. Xu, Normal Families of Holomorphic Functions and Shared Values, Israel Journal of Mathematics 129 (2002), 125-141, doi: 10.1007/BF02773159.

[6] W. K. Hayman, Meromorphic Functions, Clarendon Press, Oxford, 1964.

[7] E. Mues and N. Steinmentz, Meromorphe Functionen, die mit ihrer Ableitung Werte teilen, Manuscripta Mathematica 29 (1979), 195-206, doi: 10.1007/BF01303627.

[8] X. Pang and L. Zalcman, Normality and Shared values, Ark. Mat. 38 (2000), 171-182, doi: 10.1007/BF02384496.

[9] L. Zalcman, A heuristic principle in complex function theory, The American Mathematical Monthly 82 (1975), 813-817, doi: 10.2307/2319796.

[10] J. Schiff, Normal Families, Springer-Verlag, Berlin, 1993.

[11] W. Schwick, Sharing Values and Normality, Archiv der Mathematik 59,(1992), 50-54, doi: 10.1007/BF01199014.

[12] J. Xia, Y. Xu, Normality Criterion concerning Sharing Functions II, Bull. Malays. Math. Sci. Soc.(2) 33 (2010), no. 3, 479-486.

[13] Y. Xu, Normality Criterion concerning Sharing Functions, Houstan Journal of Mathematics 32 (2006), no. 3, 945-954. 
[14] C. C. Yang, H. X. Yi, Uniqueness theory of meromorphic functions, Science Press/ Kluwer Academic Publishers, 2003.

[15] L. Yang, Value Distribution Theory, Springer- Verlag,Berlin, 1993.

[16] L. Zalcman, Normal Families: New perspective, Bulletin of American Mathematical Society 35 (1998), 215-230, doi: 10.1090/S0273-0979-9800755-1. 
